\title{
No Evidence for an Epidemic of Stroke with the Ageing of the Population
}

\author{
Amanda G. Thrift ${ }^{a, b}$ Bin Tong ${ }^{c}$ Susana Senes ${ }^{c}$ Anne-Marie Waters ${ }^{c}$ \\ Erin Lalor ${ }^{\mathrm{d}}$ \\ aDepartment of Medicine, Southern Clinical School, Monash Medical Centre, Monash University, Melbourne, Vic., \\ ${ }^{b}$ National Stroke Research Institute, Florey Neuroscience Institutes, Heidelberg Heights, Vic., 'Australian Institute of \\ Health and Welfare, Canberra, A.C.T., and ${ }^{d}$ National Stroke Foundation, Melbourne, Vic., Australia
}

\author{
Key Words \\ Stroke $\cdot$ Hospitalisation $\cdot$ Mortality $\cdot$ Incidence $\cdot$ \\ Epidemiology
}

\begin{abstract}
Background/Aim: The ageing population in Australia may contribute to an epidemic of stroke in coming years. We aimed to assess whether deaths and hospitalisations from stroke in Australia are increasing. Methods: Nationwide ageand sex-specific hospitalisation episode data from 1996 to 1997 were used to predict future hospitalisations from stroke. Age- and sex-specific mortality data from 1997 were used to predict future mortality from stroke. These predictions were based on an expected increase in the population's age and population growth. We compared these estimates to those observed in national figures for the following 9 years. Results: Based on 1996-1997 hospitalisation rates, we calculated that hospitalisations for stroke would be expected to rise from 27,399 to 35,041 in $2005-2006$. Using 1997 mortality rates, deaths from stroke would be expected to rise from 9,126 to 12,726 in 2007. Observed hospitalisations (2005-2006) were 6,380 fewer than expected, while deaths (2007) were 4,103 fewer than expected. This represents a $2.21 \%$ annual reduction in hospitalisation rate, and a $4.0 \%$ annual reduction in mortality rate. Conclusion: Encourag-
\end{abstract}

ingly, hospitalisation and mortality rates for stroke appear to be declining. If these figures reflect actual occurrences of stroke then it may be that primary and secondary prevention strategies are working.

Copyright $\odot 2012$ S. Karger AG, Basel

\section{Background}

There is major concern in Australia that we will experience an epidemic of stroke in coming years [1]. This is based on the fact that Australia's population is ageing and stroke incidence approximately doubles with each decade of life [2].

Using stroke attack rates obtained from the first year of the North East Melbourne Stroke Incidence Study (NEMESIS), approximately 42,000 strokes were estimated to occur in Australia in 1997 [3]. Applying these same attack rates to the Australian Bureau of Statistics projected population numbers [4], stroke attack rates are expected to rise to 108,000 by 2030 [1]. Although these figures are likely to be a slight overestimate in the number of strokes, because of the greater stroke incidence in this lower socioeconomic region, this change in population demographics would result in a massive increase in the societal burden of stroke.

\section{KARGER \\ Fax +41613061234 \\ E-Mail karger@karger.ch}

www.karger.com
(C) 2012 S. Karger AG, Basel

0251-5350/12/0384-0268\$38.00/0

Accessible online at:

www.karger.com/ned
Prof. Amanda Thrift, Epidemiology and Prevention Unit Stroke and Ageing Research Centre, Department of Medicine Monash Medical Centre, Southern Clinical School, Monash University Level 1/43-51 Kanooka Grove, Clayton, VIC 3168 (Australia)

Tel.+61 39594 7567, E-Mail amanda.thrift@monash.edu 
The above stroke projections are based on three main assumptions that may not hold true. First, they are based on the assumption that age-specific stroke event rates calculated in Melbourne are representative of those found in the rest of Australia. However, even within the Melbourne region, the incidence of stroke varied [2], and so it is likely that there will be variations elsewhere in Australia. The projections are also based on the assumption that Australia's population will continue to grow in the same manner as current growth. This is unlikely, as the growth estimates using the 2001 population projections even differ from the growth estimates projected from the 2006 population [5]. Finally, future estimates of the number of strokes occurring are based on the assumption that incidence rates remain the same over time. However, it is possible that incidence rates are also declining, as has been observed in Perth, W.A., Australia [6], and other countries [7], such as the United Kingdom [8], New Zealand [9] and France [10], where stroke incidence studies have been repeated using the same methods. The decline in death rates from stroke [11] also supports the possibility that incidence is declining. Such declines in incidence may be attributable to improvements in primary and secondary prevention strategies, as has been observed in Canada and the United States $[12,13]$, while declines in mortality [13] actually reflect a better case fatality from improved treatment or a reduced severity in the strokes that are occurring. Thus, there are likely to be some inaccuracies in these estimates of the number of strokes that may occur in future years.

We aimed to assess whether there is evidence that deaths and hospitalisations from stroke in Australia are increasing. We hypothesised that the overall number of people hospitalised with stroke was increasing.

\section{Methods}

\section{National Data for Stroke}

We extracted all acute hospitalisations for stroke occurring from July 1, 1996, to June 30, 2006, from the Australian Institute of Health and Welfare's (AIHW) National Hospital Morbidity Database (NHMD), using stroke as a principal diagnosis only. Acute hospitalisations were identified as all separations for which the care type was reported as acute, new-born (with qualified days) or was not reported. Separations for other care types were excluded as were separations from psychiatric hospitals. These hospitalisations were extracted using ICD-9-CM codes 430-434 (except for those codes listed below) and 436 up to the period ending June 30, 1998 [14]. Thereafter ICD-10-AM codes I60-I64 were used [15]. These codes include acute hospitalisations as these are expected to represent new stroke events (first or recurrent). They therefore exclude the late effects of stroke and other codes not specific to an acute stroke event $(433.00,433.10,433.20,433.30,433.80,433.90$,
434.00, and 434.90). Deaths data were obtained from the AIHW's National Mortality Database, which is sourced from the Australian Bureau of Statistics and the Registrars of Births, Deaths and Marriages [16]. Stroke deaths were identified for the period from January 1, 1997, to December 31, 2007, using ICD-10 codes I60-I64 for deaths registered in those years (not deaths occurring in those years). For these deaths, stroke was recorded as the underlying cause of death, and not an associated cause.

\section{Denominators}

Australian Bureau of Statistics estimated resident population data as at December 31 were obtained for each year from 19952005. Population estimates from 2007 onwards were obtained from the Australian Bureau of Statistics population projections Series B [5]. Series B reflects the trends in fertility, life expectancy at birth, and net migration that were observed at the time of the census.

\section{Estimated Number of Deaths and Hospitalisations}

Hospitalisation episode data from the 1996-1997 financial year were used to predict future hospitalisations from stroke. Age- and sex-specific mortality data from 1997 were used to predict future deaths from stroke. These predictions were based on demographic changes to the population, i.e., an increase in the population's age and population growth [5].

\section{Comparison of Estimated with Observed Data}

We compared the estimates of projected hospitalisations and deaths from stroke with those observed in the national figures for the following 9 years.

\section{Hospitalisations and Deaths from Stroke Using a}

Population-Based Incidence Study of Stroke

Data from the North East Melbourne Stroke Incidence Study (NEMESIS), a study conducted using 'ideal criteria' for stroke incidence studies [17], was used as a comparator for hospitalisations and deaths obtained from routinely collected data. Firstly, the number of strokes occurring in Australia was estimated from the NEMESIS data by multiplying the age-specific attack rates with the population denominator for each age group. These were summed to obtain the overall number of strokes expected in Australia in 1997. The proportion of strokes that were hospitalised within the NEMESIS sample was then multiplied by the overall number of strokes to calculate the expected number of hospitalisations for stroke in Australia in the same year. The number of expected deaths in Australia was calculated by multiplying the proportions of patients within NEMESIS who died in the first 12 months of stroke by the overall number of strokes estimated. Similar calculations were undertaken to estimate the overall number of non-hospitalised deaths and the number of non-hospitalised survivors.

\section{Statistics}

Mantel-Haenszel age-adjusted rate ratios (MH-RR) were used to compare hospitalisation rates and death rates between the final observation period and the first observation period.

Ethics

NEMESIS was approved by ethics committees at each participating institution. Informed consent was obtained from participants. 


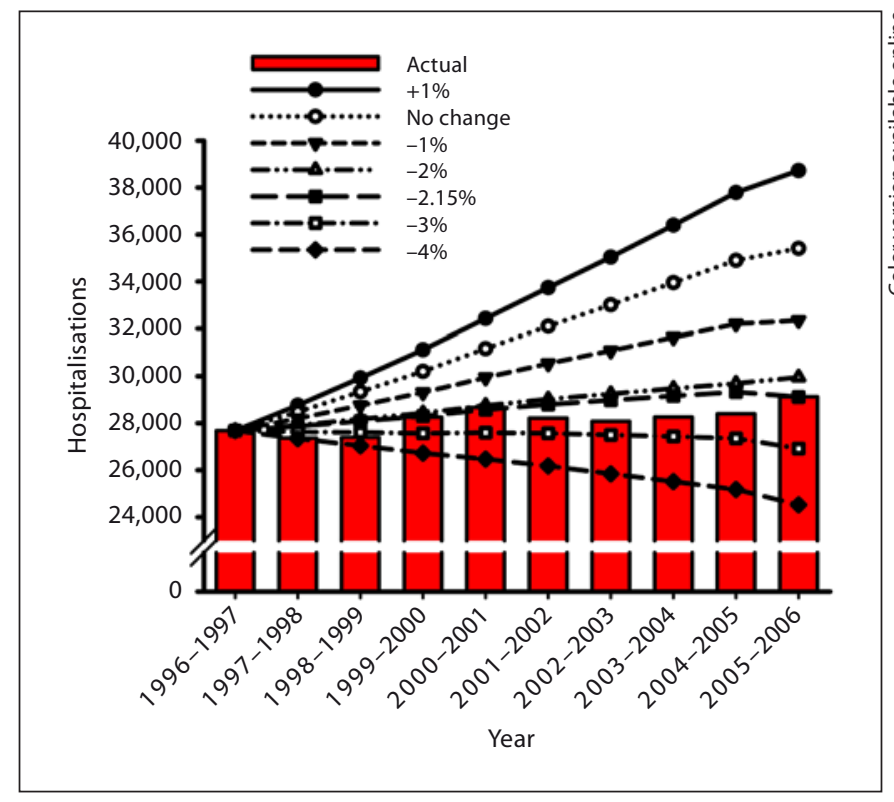

Fig. 1. Number of hospitalisations for stroke observed during 1996-1997. Each line represents a different rate of change in hospitalisations based on the financial year 1996-1997 and based on population growth and expected changes in population demographics. The red bars indicate observed hospitalisations. Color refers to the online version only.

\section{Results}

\section{Hospitalisations}

In total 27,399 hospitalisations for stroke occurred in Australia from July 1996 to June 1997. Based on the agespecific rates of hospitalisations occurring during $1996-$ 1997 and applying them to the 2005-2006 population, we calculated that hospitalisations for stroke would be expected to increase to 35,041 in 2005-2006 (fig. 1). Observed hospitalisations (2005-2006) were 6,380 (18.2\%) fewer than expected. This represents a $2.21 \%$ annual reduction in hospitalisation rate. The MH-RR for 20052006 compared with $1996-1997$ was 0.80 (95\% CI $0.79-$ $0.81 ; \mathrm{p}<0.0001)$.

The greatest reduction in hospitalisation rate for stroke occurred in the 65 - to 74 -year age group, where a $27 \%$ reduction in hospitalisation rate was observed for the period 1996-1997 to 2005-2006. The hospitalisation rate declined by $20 \%$ in the 75 - to 84 -year age group and $10 \%$ in those aged $\geq 85$ years.

\section{Deaths}

During 1997, a total of 9,126 deaths from stroke occurred in Australia. Based on the age-specific death rates

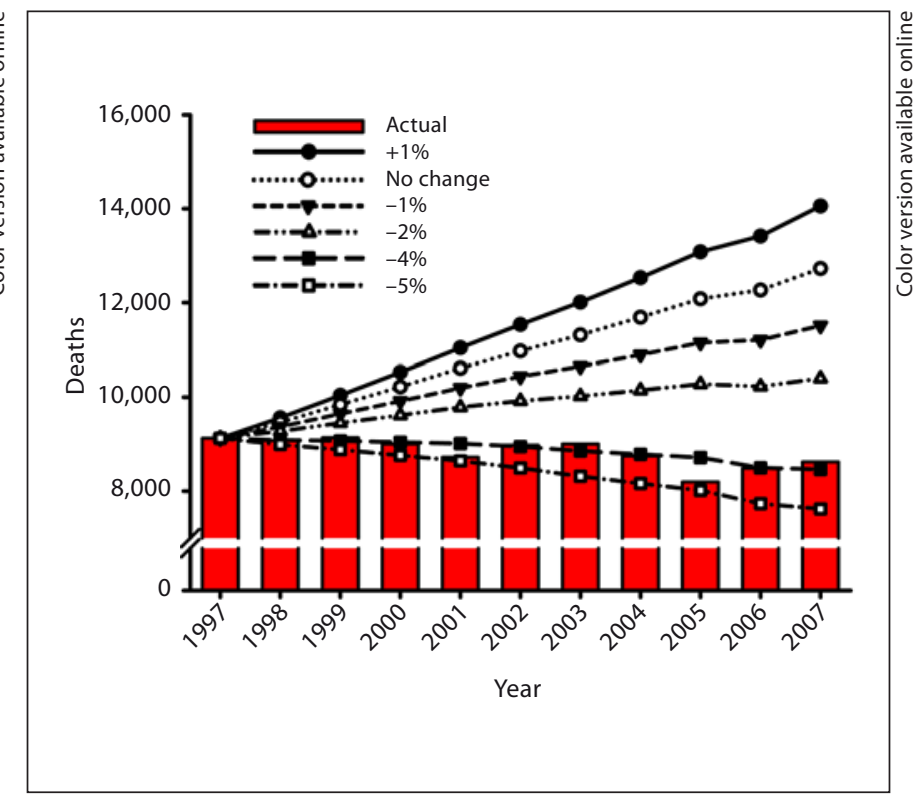

Fig. 2. Number of deaths for stroke observed during 1997. Each line represents a different rate of change in deaths based on the calendar year 1997 and based on population growth and expected changes in population demographics. The red bars indicate observed deaths during that period. Color refers to the online version only.

during 1997, we calculated that deaths from stroke would be expected to rise to 12,726 in 2007 (fig. 2). Observed deaths (2007) were 4,103 fewer (32.2\%) than expected, representing a $4.0 \%$ annual reduction in mortality rate. The MH-RR for 2007 compared with 1997 was 0.67 (95\% CI $0.65-0.69 ; \mathrm{p}<0.0001$ ).

The greatest reduction in death rate for stroke occurred in the 65- to 74-year age group (48\%). The death rate declined by $36 \%$ in the 75 - to 84 -year age group, $24 \%$ in the $\geq 85$-year age group, and $28 \%$ in the $<65$-year age group.

In-hospital case fatality also declined during the study period, declining from $17.0 \%$ in $1996-1997$ to $14.6 \%$ in 2005-2006 ( $\mathrm{p}<0.001)$.

\section{Comparison of Routine Statistics with NEMESIS}

We found that estimates of hospitalisations in Australia in 1997 obtained from NEMESIS were approximately $10 \%$ greater than those observed from routine hospital morbidity data (table 1). In addition, deaths in stroke patients within Australian hospitals estimated from NEMESIS were about $17 \%$ greater than those actually observed, and overall deaths were about $28 \%$ greater in NEMESIS than those reported through death certifica- 
Table 1. Comparison of 1997 stroke data for Australia

\begin{tabular}{lcc}
\hline & $\begin{array}{l}\text { National routine } \\
\text { statistics }\end{array}$ & NEMESIS \\
\hline Hospitalisations & 27,399 & 30,051 \\
Deaths & 9,126 & $11,711^{\mathrm{a}}$ \\
Deaths in hospital & $(4,662)^{\mathrm{b}}$ & 5,435 \\
Not hospitalised (died) & $?$ & $3,191^{\mathrm{c}}$ \\
Not hospitalised (not died) & $?$ & 1,223 \\
Total & 31,863 & 34,465 \\
\hline
\end{tabular}

Figures used to calculate totals are shown in italics. National routine data are obtained from hospitalisation and mortality data for Australia. NEMESIS figures are obtained by multiplying the age- and sex-specific attack rates for stroke (i.e., first ever and recurrent strokes combined) with the age- and sex-specific age bands for the population of Australia, and then apportioning the number hospitalised and the number who died. ${ }^{\text {a Total deaths for }}$ NEMESIS include all cases who died within 12 months of stroke, even when they died after being discharged home. ${ }^{b}$ In national mortality statistics, people who died while in hospital are counted in both the hospitalisation and the death figures listed. To avoid double counting, this figure $(4,662)$ is subtracted from the total sum of hospitalisations and deaths from stroke. ${ }^{\mathrm{C}}$ Those cases who died but would not have been counted in the hospitalisation figures as they did not attend hospital.

tions. Overall, the estimates of total strokes occurring were similar for the two sources, being approximately $8 \%$ greater in NEMESIS.

\section{Discussion}

We found that hospitalisations from stroke have not increased as expected. We also found that deaths from stroke have also not increased as expected. In contrast, both hospitalisation and mortality rates for stroke appear to be declining.

Similar to our own findings, there is evidence from Canada [18] and the United States [12, 19] that hospitalisations for stroke have been declining. There is also evidence from other regions [7-10] as well as from three repeat studies of stroke incidence conducted in Perth, Western Australia, that the incidence of stroke is declining $[6,20]$. The current figures support the notion that these declines observed in Perth may also be occurring in other parts of Australia. However, the observation of a $5.5 \%$ annual decline in incidence in Perth is much greater than the $2.21 \%$ annual reduction in hospitalisations observed in the present analysis. The fact that a greater

No Evidence for a Stroke Epidemic in Australia proportion of people with stroke were hospitalised in later time periods in Perth may, at least in part, account for some of this disparity. It is also possible that declines in the incidence of stroke may be greater in Perth than in some other major centres, and that this is the reason for the disparity in the figures observed.

The observation that declines in deaths from stroke were greater than declines in hospitalisations provides some evidence that more people with stroke are surviving the initial stroke insult. This is most likely influenced by improvements in acute stroke treatments over the past decade $[21,22]$. Another possibility is that more patients are receiving their care for stroke in a stroke care unit, as stroke units have been shown to improve outcome after stroke [23]. Although there is evidence that the number of stroke unit beds in Australia has been increasing [24], patient access to these stroke units may not necessarily mirror these increases [25]. Therefore, it is unclear whether access to stroke units has contributed to this decline in deaths following stroke.

We also found that the use of nationwide routine hospitalisation and mortality data for stroke appears to provide a reasonable approximation for the number of strokes that occurred during 1997. When interpreting these results, one must consider the differing scope and definition of hospitalisations from the AIHW NHMD and the NEMESIS and Perth studies. The hospitalisations identified in the NEMESIS and Perth studies included patients with a wide range of admitting diagnoses who were considered as potential cases and were then rigorously followed up before inclusion in the studies. In contrast, the NHMD is compiled from data supplied by the state and territory health authorities in Australia, and is a collection of de-identified electronic summary records for episodes of care in public and private hospitals in Australia [26]. Another important difference in scope is that the NHMD does not include emergency department presentations that do not require admission to hospital or episodes of non-admitted patient care provided in outpatient clinics. For the reasons outlined, the results presented here should be considered as an approximation to the number of strokes occurring in Australia rather than an exact measure. It is unclear whether this approximation would become better or worse if the pattern of stroke severity and survival changed. Further community-based studies may be warranted to better tease out the applicability of morbidity and mortality data for stroke to different settings.

There are some limitations to this study. First, there are inaccuracies in death certification for stroke [3]. 
Death certification includes those who have suffered a subdural haemorrhage, which may result in a slight overestimation of the number of deaths. However, it is more likely that a slight underestimation of deaths has occurred as the calculations are based on the underlying cause of death only. Because most people who survive the initial insult of the stroke die from other complications, the stroke is more likely to be attributed to those other causes in the mortality statistics. This may explain why the deaths attributed to stroke in the NEMESIS data are greater than those from mortality statistics. Furthermore, this attribution of deaths to other causes may have affected patterns over time as more patients are now surviving their acute event. Thus, the actual number of deaths may be underestimated and their declining pattern may be overestimated. Second, there may be inaccuracies in hospital discharge coding. For example, patients with a previous stroke may be coded as an acute stroke even though the readmission is not related to a new event. However, a recent report from the Hunter region in Australia provides evidence that the inaccuracies in hospital discharge coding may be minor [27]. Thirdly, not all people with stroke are hospitalised [28], and if the proportion of people hospitalised has changed over time, the findings of a decline in hospitalisation over time may differ from hospitalisations that are actually occurring. Data from the Perth incidence studies provide evidence that these declines in stroke hospitalisations are underestimated as the proportion of people with stroke who were hospitalised actually increased over the time period of the study [6]. Furthermore, the observation that in-hospital case fatality has also declined does not support a changing pattern of hospitalisation to those with more severe stroke. Another limitation to this study is that NHMD data are separations-based and not patient-based, so people who have more than one separation in a reference year are counted on each occasion. A separation is defined in the NHMD as an episode of admitted patient care that can be a total hospital stay from admission to discharge, transfer or death or a portion of a hospital stay beginning or ending in a change of care type (for example, from acute care to rehabilitation). We have tried to account for any double counting of acute stroke patients in the NHMD by excluding transfers from other hospitals and separations coded as statistical admission with change of care type. Further, the NHMD does not include emergency department presentations that do not require admission to hospital nor does it include episodes of nonadmitted care provided in outpatient clinics. Practices also vary across the states and territories as to whether or not a person who has a stroke and dies in the emergency department is admitted to hospital. Also, while deaths occurring in hospital for admitted patients can be identified in the NHMD, the database does not collect cause of death, so we cannot be certain that all deaths in hospital for acute stroke separations were due to the stroke. Finally, changes in classifications, coding standards, and service provision may have affected the number and rate of acute stroke separations over time.

This study provides evidence that data routinely collected on hospitalisations and mortality may provide a reasonable approximation of the actual number of strokes occurring in Australia. There is also evidence that hospitalisation rates for stroke are declining, and that mortality from stroke is declining at an even greater rate. If these figures reflect actual occurrences of stroke then, similar to Canada [13], it may be that primary and secondary prevention strategies in Australia are working.

\section{Acknowledgements and Funding}

A.T. was supported by a senior research fellowship from the National Health and Medical Research Council (NHMRC 438700). The North East Melbourne Stroke Incidence study was supported by grants from the NHMRC (project grants 154600, 307900, and 526601), VicHealth, the Foundation for High Blood Pressure Research, and the National Stroke Foundation. The National Death Index provided linkage of NEMESIS patients to ensure complete follow-up of all deaths.

\section{References}

1 Senes S: How we manage stroke in Australia. AIHW cat. No. CVD 31. Canberra, Australian Institute of Health and Welfare, 2006.

2 Thrift AG, Dewey HM, Sturm JW, Paul S, Gilligan AK, Srikanth VK, et al: Greater incidence of both fatal and non-fatal strokes in disadvantaged areas: the North East Melbourne Stroke Incidence Study. Stroke 2006; $37: 877-882$.
- 3 Thrift AG, Dewey HM, Macdonell RAL, McNeil JJ, Donnan GA: Stroke incidence on the east coast of Australia: the North East Melbourne Stroke Incidence Study (NEMESIS). Stroke 2000;31:2087-2092.

4 Australian Bureau of Statistics: Population projections (2002-2101), Australia. Catalogue No. 3222.0. 2003.
5 Australian Bureau of Statistics: Population projections (2006-2101), Australia. Catalogue No. 3222.0. 2008

6 Islam MS, Anderson CS, Hankey GJ, Hardie $\mathrm{K}$, Carter K, Broadhurst R, et al: Trends in incidence and outcome of stroke in Perth, Western Australia during 1989 to 2001: the Perth Community Stroke Study. Stroke 2008;39:776-782. 
7 Feigin VL, Lawes CM, Bennett DA, BarkerCollo SL, Parag V: Worldwide stroke incidence and early case fatality reported in $\mathbf{5 6}$ population-based studies: a systematic review. Lancet Neurol 2009;8:355-369.

8 Rothwell PM, Coull AJ, Giles MF, Howard SC, Silver LE, Bull LM, et al: Change in stroke incidence, mortality, case-fatality, severity, and risk factors in Oxfordshire, UK from 1981 to 2004 (Oxford Vascular Study). Lancet 2004;363:1925-1933.

$\checkmark 9$ Anderson CS, Carter KN, Hackett ML, Feigin V, Barber PA, Broad JB, et al: Trends in stroke incidence in Auckland, New Zealand, during 1981 to 2003 . Stroke 2005;36:20872093.

10 Wolfe CD, Giroud M, Kolominsky-Rabas P, Dundas R, Lemesle M, Heuschmann P, et al: Variations in stroke incidence and survival in 3 areas of Europe. European Registries of Stroke (EROS) Collaboration. Stroke 2000; 31:2074-2079.

11 Australian Institute of Health and Welfare: Cardiovascular disease: Australian facts 2011. Cardiovascular disease series. Cat. No. CVD 53. Canberra, AIHW, 2011.

12 Fang J, Alderman MH, Keenan NL, Croft JB: Declining US stroke hospitalization since 1997: National Hospital Discharge Survey, 1988-2004. Neuroepidemiology 2007;29: 243-249.

13 Webster F, Saposnik G, Kapral MK, Fang J, O'Callaghan C, Hachinski V: Organized outpatient care: stroke prevention clinic referrals are associated with reduced mortality after transient ischemic attack and ischemic stroke. Stroke 2011;42:3176-3182.
14 National Coding Centre 1996: The Australian version of the International Classification of Diseases, Injuries, 9th revision, clinical modification (ICD-9-CM). Sydney, University of Sydney, 1996.

15 National Centre for Classification in Health $(\mathrm{NCCH})$ : The International Statistical Classification of Diseases and Related Health Problems, Tenth Revision, Australian Modification (ICD-10-AM). Sydney, NCCH, Faculty of Health Sciences, University of Sydney, 2010.

16 Australian Institute of Health and Welfare: GRIM (General Record of Incidence of Mortality) Books. Canberra, AIHW, 2010.

$\checkmark 17$ Sudlow CL, Warlow CP: Comparing stroke incidence worldwide: what makes studies comparable? Stroke 1996;27:550-558.

18 Lewis M, Trypuc J, Lindsay P, O'Callaghan C, Dishaw A: Has Ontario's Stroke System really made a difference? Healthc Q 2006;9: 50-59.

19 Towfighi A, Markovic D, Ovbiagele B: Recent patterns of sex-specific midlife stroke hospitalization rates in the United States. Stroke 2011;42:3029-3033.

20 Jamrozik K, Broadhurst RJ, Lai N, Hankey GJ, Burvill PW, Anderson CS: Trends in the incidence, severity, and short-term outcome of stroke in Perth, Western Australia. Stroke 1999;30:2105-2111.

21 The National Institute of Neurological Disorders and Stroke rt-PA Stroke Study Group: Tissue plasminogen activator for acute ischemic stroke. N Engl J Med 1995;333:15811587.
22 Kent DM, Price LL, Ringleb P, Hill MD, Selker HP: Sex-based differences in response to recombinant tissue plasminogen activator in acute ischemic stroke: a pooled analysis of randomized clinical trials. Stroke 2005;36: 62-65.

23 Stroke Unit Trialists' Collaboration: Organised inpatient (stroke unit) care for stroke (Cochrane Review). The Cochrane Library, Issue 2, 2001. Oxford, Update Software, 2001:CD000197.

24 National Stroke Foundation: National Stroke Audit Acute Services Organisational Survey Report 2009. http://www. strokefoundation.com.au/index 2 . php? option $=$ com_docman \& task= doc_view\&gid=255\&Itemid=174, 2009 (accessed July 12,2011 ).

25 National Stroke Foundation: National Stroke Audit Acute Services Clinical Audit Report 2009. http://www.strokefoundation.com.au/ index2.php?option $=$ com_docman\&task $=$ doc view\&gid=268\&Itemid=174, 2009 (accessed July 12, 2011).

26 Australian Institute of Health and Welfare: Australian hospital statistics 2009-10. Health services series No. 40. Cat. No. HSE 107. Canberra, AIHW, 2011.

27 Marsden DL, Spratt NJ, Walker R, Barker D, Attia J, Pollack M, et al: Trends in stroke attack rates and case fatality in the Hunter region, Australia 1996-2008. Cerebrovasc Dis 2010;30:500-507.

28 Thrift AG, Dewey HM, Macdonell RAL, McNeil JJ, Donnan GA: Incidence of the major stroke subtypes. Initial findings from the North East Melbourne Stroke Incidence Study (NEMESIS). Stroke 2001;32:17321738. 